\title{
Dielectric Elastomer Grippers
}

\author{
Mills Patel, Rudrax Khamar, Akshat Shah, Tej Shah, Bhavik Soneji
}

\begin{abstract}
This paper appraisals state-of-the-art dielectric elastomer actuators (DEAs) and their forthcomingstandpoints as soft actuators which have freshly been considered as a crucial power generation module for soft robots. DEs behave as yielding capacitors, expanding in area and attenuation in thickness when a voltage is applied. The paper initiates with the explanation of working principle of dielectric elastomer grippers. Here the operation of DEAs include both physics and mechanical properties with its characteristics, we have describe methods for modelling and its introductory application. In inclusion, the artificial muscle based on DEA concept is also formally presented. This paper also elaborates DEAs popular application such as- Soft Robotics, Robotics grippers and artificial muscles.

Keyword:Dielectric Elastomer, Soft Grippers, Principle, Artificial Muscle, Soft Robotics, Electroactive Polymer, Voltage, Current
\end{abstract}

\section{NTRODUCTION}

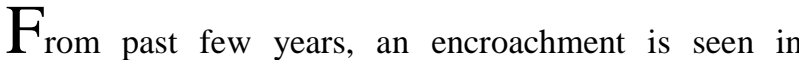
robotics, where rigid conventional robots are transcending to soft robotics with compliant structures, in this area electroactive polymers have been proposed as one of the benefactor, which is resulted in implementing it as flexible actuation mechanism. Electroactive polymer, as a classification of polymeric material that deform under electrical impulse, are appearing as auspicious replacement of current used materials for actuation and sensing application. In conflict to traditional actuator material (e.g. piezoelectric ceramics) which are better for lifting high capacity load, but small deformation, comparatively electroactive polymer are featured by their gentleness touch, flexibility, and the consequent large- deformation. Their properties resemble as artificial muscle and expected to provide soft grippers for industries and attracts attention of various robotics firm.

A simple design of DE film is shown in Fig 1. Here pair of electrode are attached to both side of film, after which electric field is transmitted through the thickness of film. The electrode used before was rigid, but in present condition it can be stretchable, which will help in gaining large area of deformation.

Manuscript received on April 27, 2021.

Revised Manuscript received on May 01, 2021.

Manuscript publishedon June 30, 2021.

* Corresponding Author

Mills Patel*, Student, Department of Mechanical Engineering, Indus University of Technology, Ahmedabad (Gujarat), India.

Rudrax Khamar, Student, Department of Mechanical Engineering, Indus University of Technology, Ahmedabad (Gujarat), India.

Akshat Shah, Student, Department of Mechanical Engineering, Indus University of Technology, Ahmedabad (Gujarat), India.

Tej shah, Student, Department of Mechanical Engineering, Indus University of Technology, Ahmedabad (Gujarat), India.

Bhavik Soneji, Assistant Professor, Departmentof Mechanical Engineering, Indus University of Technology, Ahmedabad (Gujarat), India.

(c) The Authors. Published by Blue Eyes Intelligence Engineering and Sciences Publication (BEIESP). This is an open access article under the CC BY-NC-ND license (http://creativecommons.org/licenses/by-nc-nd/4.0/)

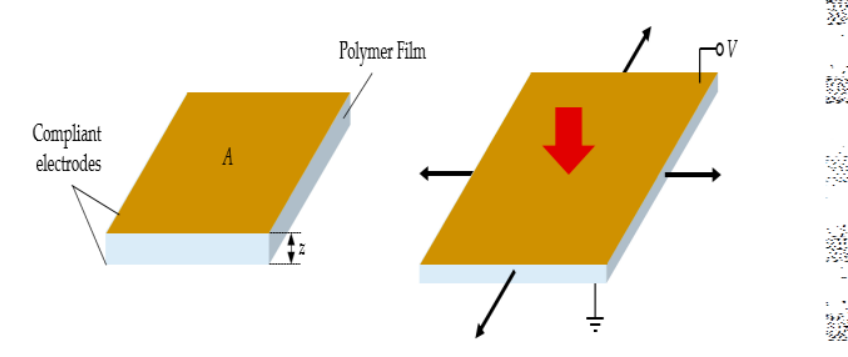

Fig1. It shows the DE operating principle, according to the voltage on/off.

In this paper, we have experimented a method to create soft grippers based on electrostatic actuation with intrinsic electro-adhesion force, which allow to control deformable, delicate objects of any dimension with controlled signal. We legitimize the method with two finger grippers as shown in figure 2 .

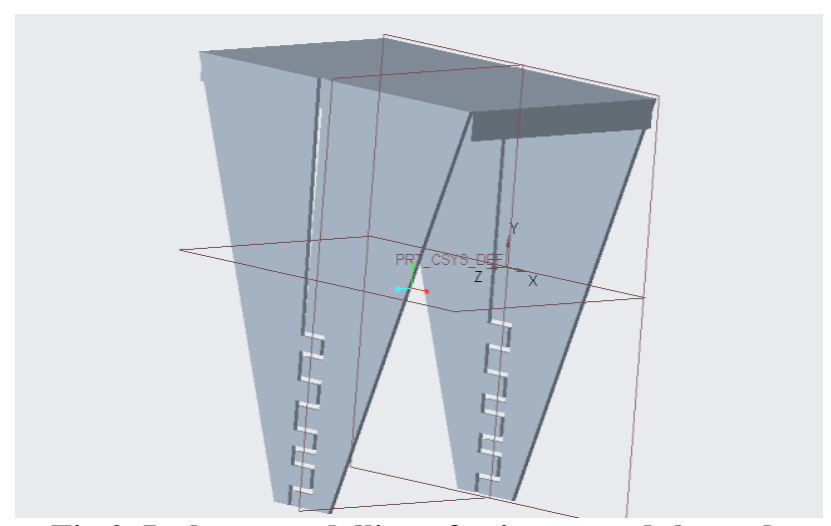

Fig 2. It shows modelling of gripper used through mechanism

It is roughly weighing around $1.8 \mathrm{~g}$, which can carry upto 400 times its weight. For instance, fragile object like egg (63 g) and comparatively a can of about (500g). Further between both the grippers a line that divides into two part is for separating positive and negative terminal. Here the gripper will be of carbon conductive paint, which will be easy to contract and relax while applying voltage to the gripper. Due to this paint a transmission of current through the acrylic polymer sheet will be uninform throughout the gripper.

This paper emphases on the review of conjectural competencies and upcoming lookout of Dielectric elastomer actuators for robotics application. This review explicates the answer of subsequent question like, why do we need indulgent robotics grippers?[6],manipulative and modelling of DE material[7], Define countless parameters at which interruption of acrylic polymer sheet (EAP) befalls.[8]

Published By: and Sciences Publication

(C) Copyright: All rights reserved.

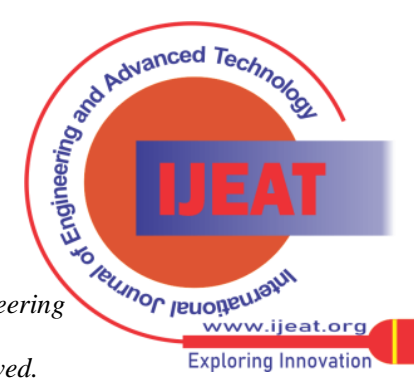




\section{RESULTS}

${ }^{[6]}$ Augmentations in soft robotics, materials science, and stretchable electronics have empoweredprecipitous progress in soft grippers. Compared to rigid grippers, end-effectors fabricated from flexible and soft components can often grasp or manipulate a larger variety of objects. Such grippers are an example of morphological computation, where control complexity is greatly reduced by material softness and mechanical compliance. Advanced materials and soft components, in particular silicone elastomers, shape memory materials, and active polymers and gels, are increasingly investigated for the design of lighter, simpler, and more universal grippers, using the inherent functionality of the materials. Embedding stretchable distributed sensors in or on soft grippers greatly enhances the ways in which the grippers interact with objects. Challenges for soft grippers include miniaturization, robustness, speed, integration of sensing, and control. Improved materials, processing methods, and sensing play an important role in future research.

\footnotetext{
${ }^{[7]}$ The actuation of DEs can be approximated as the lateral electrostatic compression and planar expansion of an incompressible linearly elastic material where the electrical

component is treated as a parallel plate capacitor. The incompressibility constraint can be expressed as

$$
\mathrm{Az}=\mathrm{P}
$$

Where $\mathrm{A}$ is the area of the electrodes, $\mathrm{z}$ is the thickness of the elastomer film between electrodes, and $\mathrm{P}$ is a constant. The stored electrical energy on the $\mathrm{DE}$ is given by the stored energy on a parallel plate capacitor.

${ }^{8}$ We conducted several experiments with and without carbon paint with various materials and dimensions to test breakdown.
}

\begin{tabular}{|c|c|c|c|c|}
\hline $\begin{array}{c}\text { SR.N } \\
\text { O }\end{array}$ & Material & $\begin{array}{c}\text { Dimension } \\
s\end{array}$ & $\begin{array}{c}\text { Breakdow } \\
\text { n Voltage } \\
\text { KV }\end{array}$ & $\begin{array}{c}\text { Breakdow } \\
\text { n Ampere } \\
\text { DC/AC }\end{array}$ \\
\hline 1) & $\begin{array}{l}\text { Acrylic } \\
\text { Sheet }\end{array}$ & $5 " \times 4 "$ & $14 \mathrm{KV}$ & $12 \mathrm{~mA}$ \\
\hline 2) & $\begin{array}{l}\text { Acrylic } \\
\text { Sheet with } \\
\text { Carbon } \\
\text { paint }\end{array}$ & $5 " \times 4 "$ & $11 \mathrm{KV}$ & $12 \mathrm{~mA}$ \\
\hline 3) & VHB 4910 & $4 " \times 2 "$ & $7 \mathrm{KV}$ & $17 \mathrm{~mA}$ \\
\hline 4) & $\begin{array}{l}\text { VHB } 4910 \\
\text { with Carbon } \\
\text { Paint }\end{array}$ & $4 " x 2 "$ & $5 \mathrm{KV}$ & $19 \mathrm{~mA}$ \\
\hline 5) & $\begin{array}{l}\text { Piezoelectri } \\
\text { c Ceramic } \\
(\mathrm{PZT})\end{array}$ & $3.5^{\prime \prime} \times 3 "$ & $6 \mathrm{KV}$ & $26 \mathrm{~mA}$ \\
\hline \multirow[t]{2}{*}{ 6) } & \multirow{2}{*}{$\begin{array}{l}\text { Piezoelectri } \\
\text { c Ceramics } \\
\text { (PZT) with } \\
\text { Carbon } \\
\text { paint }\end{array}$} & & \multirow[t]{2}{*}{$4.5 \mathrm{KV}$} & \multirow[t]{2}{*}{$25 \mathrm{~mA}$} \\
\hline & & $3.5 " x 3 "$ & & \\
\hline
\end{tabular}

\section{DISCUSSION}

Soft robotics is an emerging field with strong potential to serve as an educational tool due to its advantages such as low costs and shallow learning curves. Boneless soft robotic fingers cannot apply concentrated forces to pinch a delicate object. Dielectric elastomer actuators (DEAs) can possibly make soft grippers with better force control due to elastic actuation . A DEA is bonded on a passive layer to make a bilayer unmorphed, which can curl like a finger. The grasping of various-shaped objects is one of the most challenging issues in the robotics field. As a solution to this issue, DEAs attract a lot of attention from researchers. The inherent flexibility of DEA enables an intimate contact between an object and a gripper with a DEA, while making an efficient grasping configuration. Also, the lightweight and high energy density of a DEA are important characteristics in achieving a high weight ratio of gripper mass to grasping capability. Based on a simple working principle and large actuation strain of the DEA, different types of DE grippers have been developed with various configurations.

Dielectric elastomers, as an important category of electroactive polymers, are known to have viscoelastic properties that strongly affect their dynamic performance and limit their applications. For demonstrative purposes, a simple material law is proposed under the theoretical framework. With physically uncoupled polarization and deformation, the simple model is equivalent, to some extent, to the approach of adding Maxwell stress to the total stress in the formulation of finite-deformation viscoelasticity while leaving the inelastic stress unchanged. Boneless soft robotic fingers cannot apply concentrated forces to pinch a delicate object. A three-dimensional design of dielectric elastomer (DE) fingers with higher flexural stiffness and close to $900^{\circ}$ voltage-controllable bending for object gripping and pinching. It makes use of tension arch flexures to elevate pre-stretched DEA into a roof shape and thus magnifies the tension-induced moment, 40 times higher than a flat DEA does, to bend a stiff base frame. Such fingers make normally close-grippers to lift a payload 8-9times their weight.

Unexpected breakdown phenomena exist for DEAs. Such phenomena are derived from various factors, such as the material properties of DEAs, the compliant electrode's properties, and surface condition. To decrease the voltage level, the dielectric elastomer can be either multi-layered or processed to thin films. Another approach is the fabrication of a high voltage controller (or an amplifier) that is small and consumes low power to eliminate the hindrance in working voltage generation. According, to current scenario in Robotics Handling System there is no other product using this type of technology. It will also be capable in handling fragile objects which can be destroyed easily. With a Cheaper rate it can be available easily and helpful for medium scale industries. This is useful for society by helping and creating Human like touch Response to the objects. For use in BIONICS to GRASP VERSATILE OBJECTS in industries. and Sciences Publication

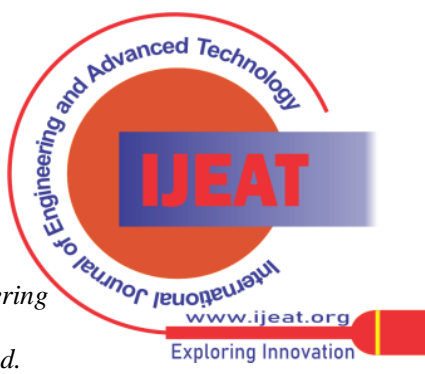


The voltage levels required to perform the actuation depend on the dielectric layer

thickness and material, and

on the need to maximize

the displacement and force output. Typically, layers of silicone 100 um thick are used. Combined with a dielectric strength of $100 \mathrm{~V} / \mathrm{um}$, a system designed as such can theoretically withhold about $10 \mathrm{kV}$. This voltage level thus became the target aimed at for the purpose of this study.

\section{WORKING OF DEAS METHODOLOGY}

Here there are two types of method for testing. One is the bilateral prestrained film where there is a circle of certain diameter and a carbon paint is done on both side of material and both side are extended with some broad line, in which one is extended from top and other from bottom. Second method is uniaxially prestrained film, where a single line is stretched on full material.
1
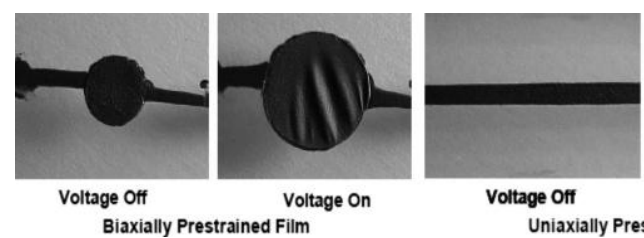

Voltage Off

Uniaxially Prestrained Film

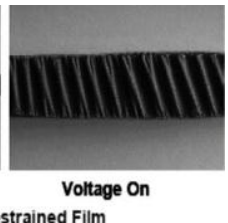

Fig 3. Actual images of fluctuation on voltage on/off.

After this a design is modelled and afterwards it is fabricated. Now here whenever an voltage will be applied to the grippers it will contract and as DC is applied it will now be able to grip the object without any current fluctuation, which helps to hold it safely to its desire position. The carbon paint here helps to stick to the object and avoid droppage of product, still the voltage is deactivated.
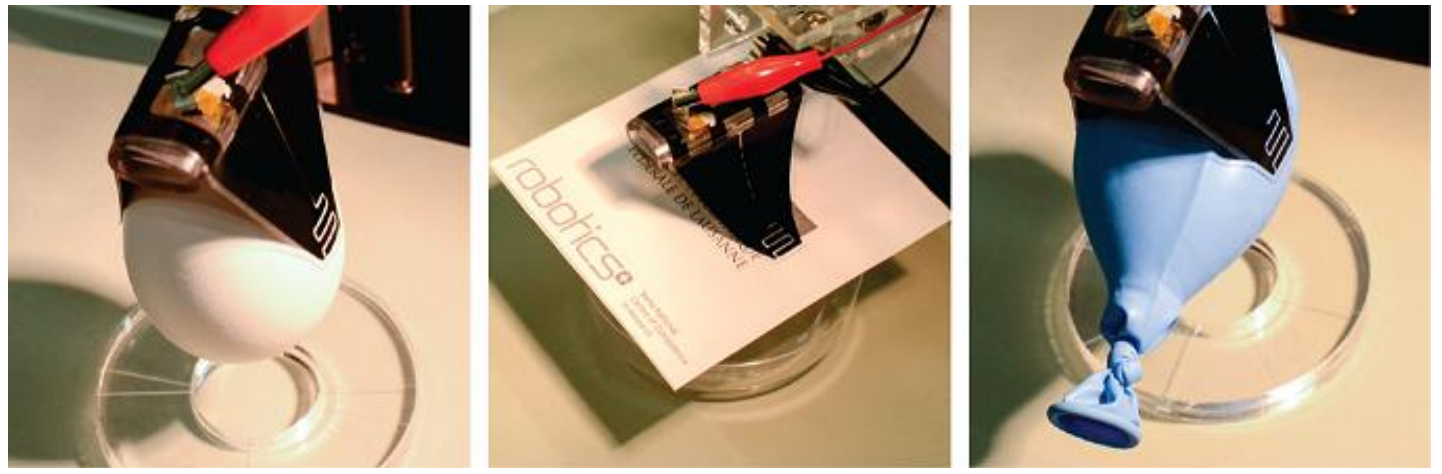

Fig 4. Concept image of DEA Grippers

\section{CONCLUSION AND FUTURE ASPECTS}

In this work, we also reported a certain breakdown voltage and current which will result in better understanding of material properties. We also tried out different design, including 4 jaw and 2 jaw mechanism. Finally we wrap-up with 2 jaw due to certain force increment in 4 jaw which has a chance to damage the fragile material.

Future studies will result in the grasping ability of soft gripper, trying out exact geometry and its properties, so that we can push this technology into industrial stage.

\section{REFERNCES}

1. Borchu, P.Pei, Q. Advances in Dielectric Elastomers for Actuators and Artificial Muscles. Macromol. RapidCommun. 2010.(https://doi.org/10.1002/marc.200900425 )

2. Dielectric Elastomer Actuator for Soft Robotics Applications and Challenges. Jihwan Park and Ki-UkKyung *Department of Mechanical Engineering, Human-Robot Interaction Laboratory, Korea Advanced Institute of Science and Technology (KAIST). Published: 16 January 2020.(https://www.mdpi.com/618968)

3. Modelling viscoelastic dielectrics Wei Hong. Department of Aerospace Engineering, Iowa State University, Ames, IA50011, USA. From ELSEVIER.

4. A Modular Reconfigurable Mold for a Soft Robotic Gripper Design.(School of Mechanical Engineering, Purdue University, West Lafayette, IN, United States, 2 Polytechnic Institute, Purdue University, West Lafayette, IN, United States, 3 College of Education, Purdue University, West Lafayette, IN, United States, 4 Department of Mechanical Engineering and Materials Science, Yale University, New Haven, CT, United States) From Frontiers Publication( https://doi.org/10.3389/frobt.2017.00046)
5. Laura, J.R.; Miguel, A.L.-M.; Raquel, V. Increasing the performance of dielectric elastomer actuators: A review from the materials perspective. Prog.Polym.Sci. 2015, 51, 188-211.( 10.1016/j.progpolymsci.2015.08.002 )

6. Tran, D.Q.; Li, J.; Xuan, F.; Xiao, T. Viscoelastic effects on the actuation performance of a dielectric elastomer actuator under different equal, un-equal biaxial pre-stretches. Mater. Res. Express 2018, 5, 065303. ( https://doi.org/10.1088/2053-1591/aac673 )

7. Dielectric elastomer fingers for versatile grasping and nimble pinching Gih-KeongLau,a) Kim-Rui Heng, Anansa S. Ahmed, and Milan Shrestha School of Mechanical and Aerospace Engineering, Nanyang Technological University, Singapore 639798 Published by AIP Publishing. (https://doi.org/10.1063/1.4983036)

8. Chuc, N.H.; Vuong, N.H.L.; Kim, D.S.; Moon, H.P.; Koo, J.C.; Lee, Y.K.; Nam, J.-D.; Choi, H.R. Fabrication and control of rectilinear artificial muscle actuator. IEEE/ASME Trans. Mechatron. 2010, 16 167-176.(10.1109/TMECH.2009.2038223 )

9. McCoul, D.; Rosset, S.; Besse, N.; Shea, H. Multifunctional shape memory electrodes for dielectric elastomer actuators enabling high holding force and low-voltage multisegmented addressing. Smart Mater. Struct. 2016, 26, 025015.( https://doi.org/10.1088/1361$665 X / 26 / 2 / 025015$ )

10. Shintake, J.; Cacucciolo, V.; Shea, H.; Floreano, D. Soft Biomimetic Fish Robot Made of Dielectric Elastomer Actuators. Soft Robot. 2018, 5, 452-465. (https://doi.org/10.1089/soro.2017.0062

11. Versatile Soft Grippers with Intrinsic Electro adhesion Based on Multifunctional Polymer https://doi.org/10.1002/adma.201504264 )

Jun Shintake , Samuel Rosset , Bryan Schubert , Dario Floreano , and Herbert Shea

12. Dielectric Elastomers as EAPs: How to Start Experimenting with Them (Springer)

Published By:

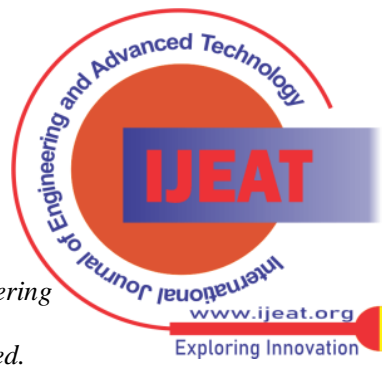


(https://link.springer.com/referenceworkentry/10.1007/978-3-319-

31530-0_34 )Herbert Shea, Soo

Jin Adrian Koh, Ingrid Graz,

and Jun Shintake

13. (https://www.epfl.ch/labs/lai/research/uh-voltage-and-energy-

recovery-for-dielectric-elastomer-actuators/ )

14. (https://www.epfl.ch/labs/lmts/lmts-research/dea/electroadhesion/ )

\section{AUTHORS PROFILE}

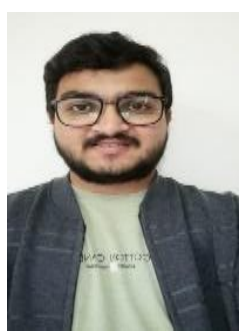

Mills Patel, is a B.Tech student of Mechanical Engineering Department of Indus University of Technology, Ahmedabad, Gujarat. During the four years of his course, he has prominently contributed in Technical fests, departmental workshops and been an enthusiastic student who seeks knowledge about the basic functioning of different mechanical machines. He was Treasurer in Indus Mechanical Engineering Association (IMEA) in 2019-20, also organised various technical and non-technical events. Managed an event on water desalination, which was an international Conference.

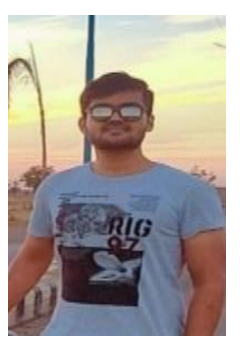

Rudrax Khamar, is a B.Tech student of Mechanical Engineering Department of Indus University of Technology, Ahmedabad, Gujarat. During the four years of his course, he has prominently contributed in Technical fests, departmental workshops and been an enthusiastic student who seeks knowledge about the basic functioning of different mechanical machines. He was President in Indus Mechanical Engineering Association (IMEA) in 2019-20, also organised various technical and non-technical events. Managed an event on water desalination, which was an international Conference.

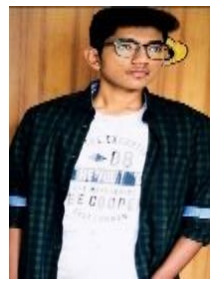

Akshat Shah, is a B.Tech student of Mechanical Engineering Department of Indus University of Technology, Ahmedabad, Gujarat. During the four years of his course, he has prominently contributed in Technical fests, departmental workshops and been an enthusiastic student who seeks knowledge about the basic functioning of different mechanical machines. He was General Secretary in Indus Mechanical Engineering Association (IMEA) in 2019-20, also organised various technical and non-technical events. Managed an event on water desalination, which was an international Conference.

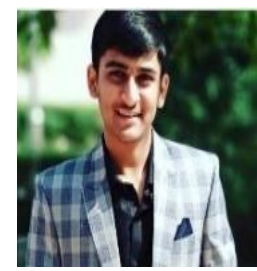

Tej shah, is a B.Tech student of Mechanical Engineering Department of Indus University of Technology, Ahmedabad, Gujarat. During the four years of his course, he has prominently contributed in Technical fests, departmental workshops and been an enthusiastic student who seeks knowledge about the basic functioning of different mechanical machines. He was VicePresident in Indus Mechanical Engineering Association (IMEA) in 201920, also organised various technical and non-technical events. Managed an event on water desalination, which was an international Conference.

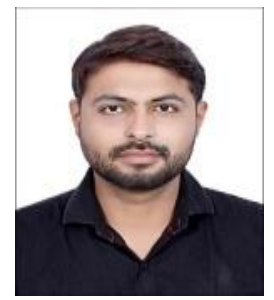

Bhavik Soneji, is a assistant professor at Indus University, Mechanical Department. Completed education in B.Tech In Mechatronics Engineering, M.Tech in Computer Aided Design \& Manufacturing. He has published 6 research paper in international journals.

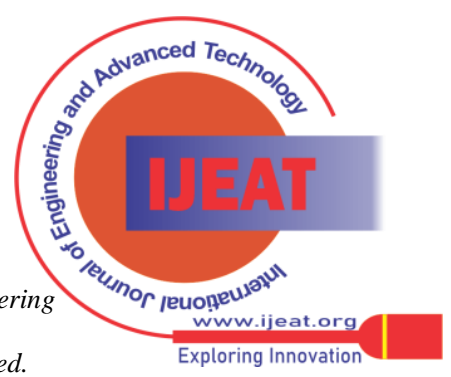

